\title{
Publisher Correction: Exosomal LINC00161 promotes angiogenesis and metastasis via regulating miR-590-3p/ROCK axis in hepatocellular carcinoma
}

\author{
Li-Na You $\cdot$ Qin-Wen Tai $\cdot$ Lin Xu $\cdot$ Yi Hao $\cdot$ Wen-Jia Guo $\cdot$ Qiao Zhang $\cdot$ Qing Tong $\cdot$ Heng Zhang $\cdot$ Wu-Kui Huang
}

Published online: 19 April 2021

(c) The Author(s), under exclusive licence to Springer Nature America, Inc. 2021

Correction to: Cancer Gene Therapy

https://doi.org/10.1038/s41417-020-00269-2

The article "Exosomal LINC00161 promotes angiogenesis and metastasis via regulating miR-590-3p/ROCK axis in hepatocellular carcinoma", written by Li-Na You, Qin-Wen Tai, Lin Xu, Yi Hao, Wen-Jia Guo, Qiao Zhang, Qing
Tong, Heng Zhang \& Wu-Kui Huang, was incorrectly published online on the publisher's internet portal on 7 January 2021 with Open Access under a Creative Commons Attribution (CC BY) license 4.0.

The copyright of the article changed on 19 April 2021 to (C) The Author(s), under exclusive licence to Springer Nature America, Inc. 2021 with all rights reserved. 